\title{
The Comparison of Hip and Knee Focused Exercises Versus Hip and Knee Focused Exercises with the Use of Blood Flow Restriction Training in Adults with Patellofemoral Pain: A Study Protocol of a Randomized Controlled Clinical Trial
}

ISSN: 2576-8875

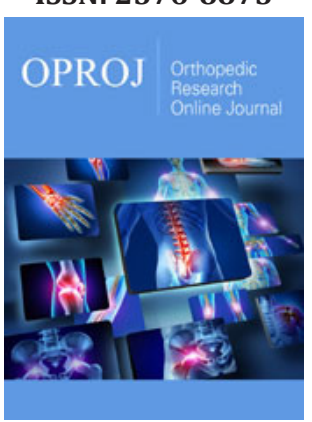

*Corresponding author: Antonis Constantinou, Department of Health Sciences, Cyprus

Submission: 酸 January 10, 2020

Published: 漹January 28, 2020

Volume 6 - Issue 3

How to cite this article: Antonis Cons, Dimitrios S, Demetris L. The Comparison of Hip and Knee Focused Exercises Versus Hip and Knee Focused Exercises with the Use of Blood Flow Restriction Training in Adults with Patellofemoral Pain: A Study Protocol of a Randomized Controlled Clinical Trial. Ortho Res Online J. 6(3). OPROJ.000640.2020.

DOI: 10.31031/OPROJ.2020.06.000640

Copyright@: Antonis Constantinou, This article is distributed under the terms of the Creative Commons Attribution 4.0 International License, which permits unrestricted use and redistribution provided that the original author and source are credited.

\author{
Constantinou Antonis ${ }^{1,2 *}$ Stasinopoulos Dimitrios ${ }^{2,3,4}$ and Lamnisos Dimitrios ${ }^{1,2}$ \\ ${ }^{1}$ Department of health Sciences, Physiotherapy Programme, European University Cyprus, \\ Cyprus \\ ${ }^{2}$ Cyprus Musculoskeletal and Sports Trauma Research Centre (CYMUSTREC) \\ ${ }^{3}$ Physiotherapy Department, University of west Attica, Greece \\ ${ }^{4}$ Laboratory of Neuromuscular and cardiovascular study of motion
}

\begin{abstract}
Background: Hip and Knee strengthening exercises are implemented in Patellofemoral Pain (PFP) rehabilitation but exercising in high loads to achieve muscle changes in strength may lead to increased patellofemoral joint stress. Low load training with Blood Flow Restriction (BFR) may allow for exercise strength benefits to proximal and distal muscles with reduced joint stress and by promoting hypoalgesia. The aim of this study is to compare hip and knee focused exercises with and without BFR training in adults with PFP for short term effectiveness.
\end{abstract}

Methods: An observer blinded RCT will be performed. We intend to include 76 volunteer participants with PFP, aged 18-40 years from Cyprus. Patients who meet the inclusion criteria will be randomised using a block number sequence in two groups: Hip and knee strengthening, BFR hip and knee strengthening. Standardized exercises will be performed under supervision of a physiotherapist three times per week for four weeks. The primary outcome measure will be the Kujala Anterior Knee Pain Scale (AKPS) at 4 weeks and 2 months follow up. Secondary outcome measures will include Visual Analogue Scale (VAS) for pain (worst and usual), quadriceps, hip extensor and abductor isometric strength, the Tampa score for Kinesiophobia and the Pain Catastrophizing Scale, as well as functional tests (shallow and deep squat) and the maximum pain free flexion angle.

Discussion: Our study results will show if there is any difference in immediate and short term outcomes in adults with PFP, regarding hip and knee strength exercises with and without BFR.

Keywords: Patellofemoral pain; PFP; Exercise; Blood flow restriction training; BFR

Abbreviations: ACSM: American College of Sports Medicine; AKP: Anterior Knee Pain; AKPS: Anterior Knee Pain Scale; BFR: Blood Flow Restriction; BMI: Body Mass Index; CKC: Close Kinetic Chain; DrG: Dr. Goniometer; DSDT: Decline Step Down Test; DVT: Deep Venus Thrombosis; HHD: Hand Held Dynamometer/Try; IBM: International Business Machines Corporation; ICC: Interclass Correlation Coefficient; IPAQ: International Physical Activity Questionnaire; ITB: Iliotibial Band; LOP: Limb Occlusion Pressure; MCID: Minimal Clinical Significant Difference; MPFFA: Maximum Pain Free Flexion Angle; MVIC: Maximum Voluntary Isometric Contraction; N: Newtons; NPRS: Numeric Pain Rating Scale; NSAID: Non-Steroidal Anti-Inflammatory Drugs; NSCA: National Strength and Conditioning Association; OKC: Open Kinetic Chain; PCS: Pain Catastrophizing Scale; PF: Patellofemoral; PFP: Patellofemoral Pain; RCT: Randomized Controlled Trial; RM: Repetition Max; SPSS: Statistical Package for the Social Sciences; TSK: Tampa Scale of Kinesiophobia; VAS: Visual Analogue Scale; VAS-U: Visual Analogue Scale-Usual Pain; VAS-W: Visual Analogue Scale-Worst Pain; CNBC: Cyprus National Bioethics Committee

Article Summary

\section{Strengths and limitations of this study}

1. This is the first study to assess the additional effects of hip strengthening with BFR training in patients with PFP in Cyprus. 
2. Alimitation of our study could be the level of blinding, but patients cannot be blinded to treatment.

3. Finally, we do not include a placebo or no treatment group due to ethical issues.

\section{Introduction}

\section{Background}

Patellofemoral Pain (PFP) is defined as "peri" or retro patellar pain aggravated by at least one of the following activities that increase stress on the patellofemoral (PF) joint (squatting, running, jumping, kneeling, stair ascent/descent, prolonged sitting) [1]. PFP is one of the most common musculoskeletal problems concerning the knee joint in young adults [2] and is considered to be a subgroup of anterior knee pain (AKP) [3]. PFP is thought to be of multifactorial origin [4] and although many factors, such as abnormal or over loading of the patellofemoral joint [5], malalignments of the lower limb and muscular imbalance of the lower extremity (local, distal or proximal) have been associated, etiology is still unknown [1]. Thus, PFP is a diagnosis of exclusion of other factors contributing to AKP and no gold standard test exists for the diagnosis [1,6-8]. Exercise is considered to be the treatment of choice according to the PFP 2018 consensus statement [9]. Exercise therapy consists mainly of quadriceps strengthening and in recent years, hip strengthening exercises have been proved appropriate [9]. Studies implementing exercise in PFP use relatively high loads, $70 \%$ of one repetition max (1RM) [10-16]. When targeting the quadriceps, this can increase patellofemoral joint stress and potentially aggravate patients symptoms, leading to a reduction in exercise load to avoid pain and subsequently strengthening parameters as set by the American College of Sports Medicine (ACSM) are not met [17].

Blood Flow Restriction training (BFR) is a method of exercise where the limbs blood flow is restricted by a pneumatic cuff reducing arterial blood flow and venous return. This technique forces the musculature to exercise in an oxygen deprived environment leading to early fatigue [18]. While exercising with low loads of $20-30 \%$ of $1 \mathrm{RM}$ using BFR, muscle hypertrophy is promoted and well as strength, compared to the same program without BFR $[19,20]$. It is thought that these changes occur due to a greater accumulation of metabolites, due to both increased production in the more hypoxic state and their limited removal due to the BFR itself [21]. Moreover, it has been shown that exercise with BFR could induce hypoalgesia in patients with AKP $[22,23]$ although mechanisms are still unclear. Interestingly, not only muscles distal to the cuff but proximal muscles above the cuff, have also been hypothesized to benefit from BFR training [24]. Taking into consideration the above factors, BFR training could facilitate in PFP rehabilitation, by allowing exercise benefits with reduced PF joint stress and by promoting hypoalgesia. Studies on AKP patients have shown positive results in hypoalgesia $[22,25]$ There is one study that used BFR training for PFP patients but did not include hip exercises [23]. This will be the first study to compare hip and knee focused exercises with and without BFR training in adults with PFP.

\section{Specific aims and hypothesis}

The purpose of this study is to compare hip and knee focused exercises with and without BFR training in adults with PFP. The main outcome of this study is function ability which will be measured with the Kujala Anterior Knee pain Scale translated in the Greek language at four weeks post intervention and at two months follow up. Our null hypothesis is that there will be no difference between groups for primary and secondary outcomes measured at four weeks and two months post intervention.

\section{Methods and Analysis}

\section{Trial/Design}

This a randomized controlled clinical trial with first primary end point at four weeks and second primary end point at two months (Figure 1). Methods of this study follow the SPIRIT reporting guidelines [26].

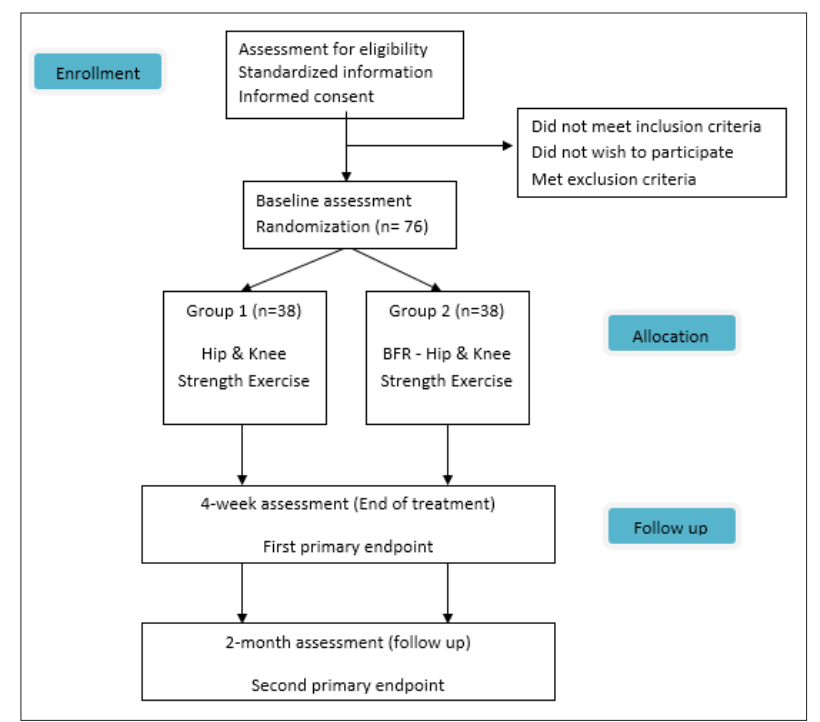

Figure 1: Patient flow through the study. 


\section{Sample size}

Sample size was determined through power analysis. The Minimal Clinically Significant Difference (MCID) of our main outcome measure, Kujala Anterior Knee Pain Scale, was used for the estimation. The MCID of the Kujala scale has been reported to be 10 points [26] with an estimated standard deviation of 13,5 points [27]. For a power of 0.80 and a level of significance $a=0.05$ and considering a two tailed t-test the sample size was calculated to be 60 participants total (30 in each group). The GPower 3.0.10 software was used to calculate the sample size. By estimating a possible $20 \%$ drop out we concluded to a sample size of 75 . To even out the two groups finally we conclude to 38 participants in each group.

\section{Participants}

The participants of this study will be volunteers and considered a sample of convenience since no official records or data base exists in Cyprus with all PFP patients. Participant recruitment will begin in November 2019. Potential participants will be informed for the study through printed posters around the European University Cyprus (EUC) campus, local social media online posters and will volunteer for inclusion. Screening will be carried out by an orthopedic surgeon (P.X.), at the musculoskeletal laboratory of the EUC to determine their eligibility based on the inclusion and exclusion criteria described below.

\section{Inclusion criteria}

Volunteers to be included can be either male or female, must be 18-40 years of age and should have at least a 4 week [27], history of peri or retro-patella non traumatic pain, with intensity of worst pain during the previous week at $3 \mathrm{~cm}$ on the Visual Analogue Scale (VAS). Pain should be aggravated by at least two of the following functional tasks: squatting, kneeling, prolonged sitting, stair ascending or descending, hopping or running. During physical examination pain must be present with either palpation of the patella facets or with the patella compression test or a deep squat. Volunteers with bilateral symptoms will be documented but the limb with the worst pain will be used for analysis. Inclusion criteria are based on latest PFP guidelines [1].

\section{Exclusion criteria}

Athletes will be excluded, as well as participants with a high level of physical activity based on assessment of the International Physical Activity Questionnaire (IPAQ) in Greek [28]. This will be performed on the day of initial screening. Their exclusion is based on the fact that they may not respond to the level of intensity of the exercises provided in this study and for homogeneity of the sample. Volunteers with the following characteristics will also be excluded: History or current meniscus ligament or other knee injury and/or surgery. Other knee pathology such as knee osteoarthritis, OsgoodSchlatter or Sinding-Larsen-Johanssen syndrome or tendinopathy of muscles surrounding the knee. Knee instability, feeling of "giving way", history of subluxation or dislocation of the knee joint or joint edema. Extended use of NSAID or cortisone. Referred pain from lumbar spine or another region. Patella dysplasia, rheumatoid arthritis or neurological syndromes or diseases [1,16]. During clinical examination volunteers with pain located on the patella tendon that is eliminated with isometric contraction, the pes anserinus, the Iliotibial Band (ITB), or with a positive medial or lateral patella apprehension test will also be excluded [29]. The above exclusion criteria are used to avoid any subgroups of PFP and/or other causes of anterior knee pain. Volunteers that had previous treatment for PFP in the past 6 months will also be excluded to avoid non responders and carryover effects from previous treatments. Furthermore, volunteers with unexplained chest pains, cardiovascular disease, renal disease, vascular surgery or disease, Deep Venous Thrombosis (DVT) or high risk for DVT, resent surgery $\leq 6$ months, high blood pressure $(\geq 140 / 90 \mathrm{mmHg}$ ), dizzy spells, history of fainting or dizziness with exercise, pregnancy and with contraindication to exercise will also be excluded [23]. The above criteria intend to exclude people that cannot participate in an exercise program or use BFR training.

\section{Randomization}

Volunteers who meet inclusion criteria will receive oral and written information and give informed consent to participate in the study by signing a consent form. They will be randomized into one of two treatment groups. Group 1 will be the Hip and Knee Exercise group and Group 2 the BFR training Hip and Knee exercise group. Randomization will be carried out using a block sequence of 4 by an external statistician (DL). Block number sequence will be generated by the statistician using https://www.sealedenvelope. com [30]. After the volunteers initial screening and consent, the statistician will contact each participant via telephone and assign them to their treatment group. This way it is ensured that the assessor (AC) will be blinded to participants intervention group. The block randomization method was selected in order to maintain a balance in sample size across groups over time [31].

\section{Interventions}

All interventions will take place in the musculoskeletal laboratory and the training center lab of the EUC. Time consistency in treatments will be a goal, in order to train participants at similar circadian rhythm. Room temperature for both labs will be set at $25{ }^{\circ} \mathrm{C}$. Two experienced physiotherapists will each be randomly assigned to supervise one of the two intervention groups and provide the treatment. The therapists role is to supervise treatments and their progression, keep track of compliance with the study design, adverse effects and drop outs. If patients do not comply with the study (seek other treatment for PFP, exercise during the study, inability to attend treatments) design they will be excluded. The two therapist will be blinded as to the other groups treatment protocol and the studies outcomes. They will be given written information for all procedures of their intervention group in order to ensure consistency throughout the study. Data collected by the therapists will be stored at the EUC laboratories and will only be available to them. Additionally, the therapists will be assisted by fourth year physiotherapy undergraduate students in supervising participants during treatments. Description of interventions are summarized in Table 1. Participants who fail to complete at least 
10/12 therapy sessions will be excluded and their data will not be analyzed. All therapy sessions will be supervise by a physician (GL) who will be in charge of participants safety. Any adverse events will be reported with publication of final results. Participants are free

Table 1: Treatment protocols performed in the study. to withdraw from the study at any given moment. In case of any complaints, participants have the right to file official complaints to the university bioethics committee.

\begin{tabular}{|c|c|}
\hline Group 1 (Hip and Knee Exercise Program) & Parameters (Dose) \\
\hline $\begin{array}{c}\text { Hip abduction with ankle weights (side-lying)* } \\
\text { Hip extension (machine)* } \\
\text { Hip abduction against elastic band (standing)† } \\
\text { Hip lateral rotation against elastic band (sitting) } \dagger \\
\text { Seated knee extension }\left(90^{\circ}-45^{\circ}\right) \text { (sitting)* } \\
\text { Prone knee flexion* } \\
\text { Squatting }\left(0^{\circ}-45^{\circ}\right)^{*} \\
\text { Single Leg press }\left(0^{\circ}-45^{\circ}\right) \text { (machine)* }\end{array}$ & $\begin{array}{c}* 3 \text { sets of } 10 \text { rep } \\
70 \% \text { of } 1 \mathrm{RM} \\
(1: 2) \text { con/ecc } \\
30 \text { sec rest / sets } \\
2 \text { min rest / exersices } \\
+3 \text { sets of } 10 \text { rep } \\
\text { At } 10 \mathrm{RM} \\
(1: 2) \text { con/ecc } \\
30 \text { sec rest / set } \\
2 \text { min rest / exercise }\end{array}$ \\
\hline Group 2 (BFR - Hip and Knee Exercise Program) & Parameters (Dose) \\
\hline $\begin{array}{c}\text { Hip extension (machine) })^{* *} \\
\text { Hip abduction (machine) })^{* *} \\
\text { Seated single knee extension (machine) })^{* *}\left(90^{\circ}-45^{\circ}\right) \\
\text { Single Leg press }\left(0^{\circ}-45^{\circ}\right)(\text { machine })^{* *}\end{array}$ & $\begin{array}{c}\text { **1st set 30reps followed by } 3 \text { sets of 10reps } \\
30 \% \text { of } 1 \mathrm{RM} \\
(2: 2) \text { con/ecc } \\
70 \% \text { LOP } \\
30 \text { sec rest / sets } \\
\text { 3min rest / exersices }\end{array}$ \\
\hline Co-intervention Assisted, Passive, Static Stretching: & Parameters (Dose) \\
\hline $\begin{array}{l}\text { Hamstrings \& plantar flexors } \\
\text { Quadriceps } \\
\text { Iliotibial band }\end{array}$ & 3rep. of $30 \mathrm{sec}$ \\
\hline $\begin{array}{l}\text { Abbreviations: rep. - repetitions, RM - Repetition Max, sec. - seconds, } \\
\text { con - concentric, } \\
\text { ecc - eccentric, LOP - Limb occlusion pressure }\end{array}$ & \\
\hline
\end{tabular}

\section{Group 1 (Hip and knee exercise group)}

Participants will follow a supervised hip and knee strengthening exercise protocol with a combination of stretching, 3 times a week for 4 weeks to a total of 12 sessions. Therapy sessions will be executed in group form. Exercises will focus on strengthening the hip extensors, abductors and external rotators, as well as the quadriceps and hamstrings in both open and Closed Kinetic Chain (CKC). Open Kinetic Chain (OKC) exercises will be executed first followed by CKC exercises. Exercises utilizing ankle weights or mechanical resistance will be set at a load of $70 \%$ of $1 \mathrm{RM}$ and 3 sets of 10 rep. will be carried out for each exercise. Elastic resistance exercises will be standardized to the maximum resistance the participant can execute 10RM for 3 sets. The time under tension and tempo of the exercises will be set by the Metronome app installed on a Smartphone. The tempo will be set at 1:2 (concentric/ eccentric) at $60 \mathrm{bpm}$ with auditory cue. Rest between sets will be $30 \mathrm{sec}$ and between exercises $2 \mathrm{~min}$. Pain with exercise will not be permitted. If pain exists, then resistance will be adjusted and documented. Exercise progression will be individualized based on weekly assessments of RM for each exercise by the groups physiotherapist. These assessments will be performed at the beginning of each week. The duration of each session is estimated to be approximately $45 \mathrm{~min}$, with the exception of the first session of each week which is estimated to be $80 \mathrm{~min}$ due to the addition of strength testing.

\section{Group 2 (BFR-training hip and knee exercise group)}

This group will follow a hip and knee strengthening exercise protocol using BFR training with a combination of stretching, 3 times a week for 4 weeks to a total of 12 sessions. BFR cuff (Sports Rehab Tourniquet(C) will be used, with a width of $10 \mathrm{~cm}$ and a length of $116 \mathrm{~cm}$ or $84 \mathrm{~cm}$ depending on the diameter of the participants thigh. The length of the cuff should be adequate to wrap around the upper third of the participants thigh and the edges of the cuff should overlap as not to leave a gap between them. Therapy sessions will be supervised and in groups. Exercises will focus on strengthening the hip extensors and abductors, as well as the quadriceps both in open and closed kinetic chain. OKC exercises involving the hip musculature will be executed first followed by quadriceps strengthening and CKC exercises. Exercises will be carried out using mechanical resistance and will be set at a load of $30 \%$ of ORM with a limb occlusion pressure (LOP) of 70\%. Four sets will be carried out for each exercise. The first set will consist of 30reps. and the next 3 sets of 15 reps. for each exercise. The time under tension and tempo of the exercises will be set by the 
Metronome app installed on a Smartphone. The tempo will be set at 2:2 (concentric/eccentric) at $60 \mathrm{bpm}$ with auditory cue. Rest between sets will be $30 \mathrm{sec}$ and between exercises $2 \mathrm{~min}$. Note that the cuff will stay inflated during the 30 sec rest but will be deflated during the resting period between exercises and re-inflated at the beginning of the next exercise. Pain with exercise will not be permitted. If pain exists, then resistance will be adjusted and documented. Exercise progression will be individualized based on weekly assessments of RM for each exercise by the groups physiotherapist. Assessments will be performed at the beginning of each week. The duration of each session has been estimated to be approximately $60 \mathrm{~min}$. including LOP estimation, with the exception of the first session of each week that is estimated to be $80 \mathrm{~min}$ due to the addition of strength testing.

\section{Estimation of 1RM using the 5RM test}

One repetition max (ORM) is defined as the maximum amount of weight that a person can lift completing 1 repetition [32]. We chose to estimate the 1RM through the 5RM test as it is a valid way of estimating ORM [33] with less resistance and thought to be more tolerable for patient population. Muscle testing will be unilateral for the involved limb and assessment will include single leg press 0o-60o, leg extension 90o-45o, hip abduction and extension using Technogym $®$ equipment mechanical resistance and using ankle weights and Thera Band Professional Non-Latex Resistance Bands for elastic resistance exercises. Before testing, participants will warm up using a stationary bicycle $(1 \mathrm{~W} / \mathrm{kg}$ Body weight, at $\approx 60 \mathrm{rpm}$ ) for $5 \mathrm{~min}$. The order of testing will be standardized, first at the leg press, then the multi hip machine for hip extensors and hip abductors and finally on the leg extension machine for the quadriceps. For ankle weights and elastic bands hip muscles will be tested prior to knee musculature. Pain will be allowed during testing but should not exceed a level of 2/10 on a NPRS. After a 3 min rest the participant will initiate the trial with an estimated resistance of about $50 \%$ of their estimated maximum and complete 10 repetitions for familiarization and warm up. After a one-minute rest the resistance will be adjusted, the test repeated for 6reps. until the participant is able to complete five-repetitions but not 6 . Twominute rests are to be left between maximal tests. Once the $5 \mathrm{RM}$ is measured the $1 \mathrm{RM}$ can be calculated as the $5 \mathrm{RM}$ is set to be $87 \%$ of 1RM. For example, if the weight lifted for 5 reps. max was $87 \mathrm{Kg}$ then the $1 \mathrm{RM}$ would be $100 \mathrm{~kg}$. The $5 \mathrm{RM}$ should be estimated within 3 to five trials. These testing procedures are within the guidelines of the National Strength and Conditioning Association (NSCA) [34].

\section{RM test}

The 10RM test is conducted in the same manor with the 5RM test. The difference is that the goal is to estimate the 10RM and so the initial resistance is set for the participant to achieve a 20rep. warm up. These testing procedures are within the guidelines of the National Strength and Conditioning Association (NSCA) [34].

\section{Estimation of Limb Occlusion Pressure (LOP)}

Participants LOP will be estimated at the beginning of each session for all exercise positions; standing, sitting and semi seated.
We chose to estimate LOP daily to ensure accurate measurements as LOP changes based on body position [35], and the circadian cycle, in case a participant will not be able to attend sessions at the same time each day. LOP measurements will be assessed by the groups physiotherapist before each session in the following way and order. The participant will be placed in standing position with the BFR cuff placed on the most proximal third of the thigh. The participant will rest for three minutes before assessment commences. The therapist will palpate the posterior tibial artery and by using a Edan SD3 Vascular Doppler W/ 8MHZ the artery will be located. This will be verified by an auditory signal from the Doppler. Then, the cuff will be inflated steadily in increments of about $10 \mathrm{mmHg}$ until the auditory signal is completely lost. This pressure is recorded as the participants LOP. Following the standing position, the next measurement is taken in the seated and finally in the semi-seated position. The cuff will be inflated at $70 \%$ of LOP during exercise.

\section{Co-interventions}

Both groups will receive stretching of the hamstrings, plantar flexors, quadriceps and Iliotibial Band (ITB) in the same manor at the end of each session. Passive stretching will be executed by the physiotherapist with $3 \times 30$ sec stretch for each muscle group. Hamstrings and plantar flexors will be stretched with the patient in the supine position and the therapist flexing the hip with a straight leg raise while bringing the foot in dorsiflexion. The quadriceps will be stretched with the patient in the side-lying position and the therapist will extend the hip with the knee in flexed position, while stabilizing the pelvis. To stretch the ITB from the same position the therapist will let the extended hip to drop into adduction $[10,11]$. Besides stretching no other co-interventions will be allowed. No home-based exercises will be given and participants will not be allowed to exercise for the duration of the study. Participants will be allowed to take pain medication if prescribed by their personal doctors but will be instructed to stop 24 hours prior to assessments.

\section{Outcome assessment}

Assessments: All eligible participants will go through an initial assessment to determine baseline clinical characteristics, somatometric and demographic characteristics, as well as baseline level of all outcome measures. Baseline measures include, age, sex, weight reported in kg using a typical weight scale, height in meters using a fixed measure preset in the lab, estimation of Body Mass Index (BMI) with the formula BMI=weight/height 2 in $\mathrm{Kg} / \mathrm{m}^{2}$. Symptom duration of the involved limb and all following outcome measures. All measures will be assessed by one assessor (AC), an experienced physical therapist with more than 10 years of experience, at baseline, 4 weeks and at 2 months follow up. All data will be recorded and stored at the EUC and will only be available to the research teams assessor. After the baseline assessment the participants data will be electronically documented and each participant will be assigned a unique code number (ex 001, 002, 003 etc.). This will ensure the assessor is blinded to patient personal data during analysis and as mentioned before, allocation will be pre-set by an external statistician (DL) and the assessor (AC) will be blinded to participants treatment group. 
Primary outcome measure: The primary outcome measure for our study is functional limitation related to symptoms. For this we chose to assess functional limitations with the Kujala Anterior Knee Pain Scale (AKPS) [36]. The AKPS is a 13-item questionnaire that documents response to six different activities such as walking, running, jumping, stair climbing, squatting and prolonged sitting with knee bent, as well as patients symptoms, such as limping, inability to weight bear on the affected limb, swelling, abnormal patellar movement, muscle atrophy and limitations of flexion of knee joint. The AKPS asks about the duration of symptoms and limb(s) affected. The maximum score is 100 (no pain/disability/limitation) and the minimum 0 (worst possible function). This questionnaire has been previously used in PFP studies and is found to be a valid and reliable measure for PFP patients with a minimally clinical significant difference (MCID) of 10points [37]. The Greek version has been found to have good internal consistency (Cronbach's $\mathrm{a}=0.942$ ), test-retest reliability (ICC $=0.921$ ) and concurrent validity $(r>0.7)$ [38].

\section{Secondary outcome measures}

Pain: Pain will be assessed using the visual analog scale VAS $0-10 \mathrm{~cm}$. This scale is a $10 \mathrm{~cm}$ horizontal line with two small vertical lines at the two ends. The far-left side is 0 "no pain" and the far right is 10 "worst pain". Participants will be asked to mark with a horizontal line to rate the level of worst pain VAS-W and the level of their usual pain VAS-U that they experienced during the course of the previous week. A standard ruler will be used to measure the distance from 0 to the participants mark. The VAS scale measuring these two types of pain have been shown to be valid and reliable in patients with PFP with an MCID of $2 \mathrm{~cm}$ [37].

Pain with single leg squatting (shallow-deep): Two depths of squatting will be assessed and the VAS scale will be used to quantify the level of pain with each squat. For the first Single-leg squat (shallow): the participant will stand in front of the treatment bed and the height of the bed will be adjusted to be the mid-point of his thigh (halfway between the greater trochanter and popliteal fossa midpoint). For the second Single-leg squat (deep): the beds height will be adjusted to the popliteal fossa midpoint. Participants will perform the squat with hands crossed over the chest until they touch but not sit on the beds surface. At the end of each test, the VAS scale will be used to measure pain felt during the squat. This testing method has been previously used to assess pain in anterior knee pain patients [39].

Psychometric evaluation of pain: Kinesiophobia and Catastrophizing have been found to be elevated in patients with PFP and have been found to be related to reduced levels of physical activity and pain $[39,40]$. In addition, it has been proposed that the assessment of function alone with the AKPS may not be sufficient as many patients with PFP may be avoiding strenuous activities and score higher on functional outcome scales. Thus, we chose to assess the levels of Kinesiophobia and Catastrophizing of our participants using the Tampa Scale of Kinesiophobia (TSK) and the Pain Catastrophizing Scale (PCS). These scales have been used previously for the PFP population $[39,40]$.
Kinesiophobia: The Tampa scale of Kinesiophobia (TSK) was originally constructed by Kori et al. [41]. We will assess Kinesiophobia using the Greek Version of the TSK that has been found to be valid and reliable [42]. This scale consists of 17 items that assess fear of injury due to movement. Participants will be asked to make ratings of their degree of agreement for each of the 17 items. Ratings range from 1 (strongly disagree) to 4 (strongly agree). Responses will be summed after reversing the scores of items 4,8,12 and 16 . Higher total scores relate to higher related fear.

Catastrophizing: The Pain Catastrophizing Scale (PCS) assesses the cognitive process by which pain is seen as an extreme threat and from which the patient suffers exaggerated negative consequences [43]. We will assess catastrophizing using the Greek Version of the PCS that has been found to be valid and reliable [44]. The questionnaire consists of 13 items (statements) describing pain experience and participants are asked to indicate whether they agree with these statements on a five point scale rating from 0 (not at all) to 4 (always). Scores are summed to calculate a final score. A high score indicates a high level of pain catastrophizing. The PSC measures three categories: rumination, magnification and helplessness. Rumination refers to the patients' inability to apart the pain from his mind, magnification expresses the exaggerated cognitions of pain as a threat, and hopelessness is the estimation that the patient cannot do anything to influence his pain [43].

\section{Functional test}

Maximum pain free flexion angle using the decline step down test: The Decline Step Down Test (DSDT) is a functional test recently developed [45]. Its purpose is to measure the Maximum Pain Free Flexion Angle (MPFFA) of the knee joint whilst descending a $20 \mathrm{~cm}$ high step with a surface inclination of $20^{\circ}$. For this test the participant will be asked to step down from a custom made step with a height of $20 \mathrm{~cm}$ and an inclination of $20^{\circ}$ bringing the uninvolved limb forward and to the ground while leaving the involved limb on the step. The participant must descend to the maximum point where no pain is present. To ensure no pain, the assessor will ask the participant to rate any pain on a numeric pain rating scale 0-10 during the procedure. At this point the assessor will take a photo using the Dr. Goniometer (DrG) app, installed on an iPhone 6S. Using the app, the assessor will measure the MPFFA. This test has been developed for patients with PFP and is thought to achieve a better flexion angle than the normal step down test, as the ankle in this test is in plantar flexion and any restriction in range of motion from the ankle joint will not affect the knee range of motion during the decent. The test has been found to be reliable with intra-observer reliability ICC $=0.83$ and inter-observer reliability ICC $=0.85$. It has been shown to be valid as it has been correlated with the AKPS ( $\mathrm{r}=0.31, \mathrm{p}=0.030)$ [45].

Strength: Strength tests will measure isometric Maximum Voluntary Contraction (MVIC) with the use of a MicroFET2 ${ }^{\mathrm{TM}}$ Hand Held Dynamometer (HHD). Strength tests will not be used to assess differences between involved and uninvolved limbs as patients with bilateral symptoms are eligible for this study. Testing will help identify any changes in MVIC strength of our participants 
comparing baseline measures to follow up measures. We will assess the MVIC of the quadriceps at $\approx 60$ o of knee flexion from the seated position with the dynamometer placed on the anterior aspect of the tibia, $5 \mathrm{~cm}$ above the lateral malleolous. Hip extensors will be assessed from the prone position with the dynamometer placed on the posterior aspect of the tibia, $5 \mathrm{~cm}$ above the lateral malleolous as well as hip abductors in the side-lying position with the hip abducted to $\approx 10^{\circ}$ with the dynamometer placed laterally, $5 \mathrm{~cm}$ above the lateral malleolous. These muscle groups were selected as they are the main muscle groups involved in our exercise programs. Strength will be recorded in Newtons (N). Testing will begin with 2-3 sub-maximal contractions against the dynamometer for familiarization and warm up followed by a maximal isometric contraction of a $5 \mathrm{sec}$ duration with standard verbal encouragement by the assessor. The participant will rest for $30 \mathrm{sec}$ and the test will be repeated. If the two measures differ less than $10 \%$ then no other testing will follow. The mean of the two measures will be used for analysis. If measures differ greater than $10 \%$, tests will be repeated until the above criteria is met [46]. Placement of the dynamometer will be marked with a marker to ensure consistency between trials.

Planed statistical analysis: Statistical analysis will be performed by the studies assessor (AC) who is blinded to the participants treatment group using the IBM SPSS "Statistical Package for the Social Sciences" (SPSS, Version 20.0). Descriptive statistics will be analyzed with the calculation of means, standard deviations, minimum and max values for each variable. A normality check will follow using the One Sample Kolmogorov-Smirnov test $(\mathrm{p}<0.05)$. The chi-squared test will be used to test for difference between the two groups with respect to the categorical variable gender. To check for differences within groups (baseline-4weeks, baseline-2months follow up and 4weeks-2months follow up) we will use the paired t-test for each comparison. In case of nonparametric data, the Wilcoxon signed rank test will be used. In order to estimate differences between the two groups (baseline-4weeks, baseline-2months follow up and 4weeks-2months follow up) we will use the mixed two way ANOVA with time as a within-subjects factor and treatment as the between subjects factor. Homogeneity of variance will be checked with the Levene test $<0.05$. In the case of not normally distributed data we will use the generalized mixed effect model with treatment as a fixed factor, time as a random factor, and inverse as the link function. Possible correlations will also be checked for our outcome measures using the Pearson correlation coefficient. In case of not normally distributed data the Spearman correlation coefficient will be used if there seems to be a linear correlation from a scatter plot. Missing values in the Patient Reported Outcome Measures will be imputed using Multiple Imputation (MI) [47]. Only upon agreement by the authors that there will be no further changes and/or analysis, results of the study will be published.

No patient or public involvement: This study was designed without patient involvement. Patients were not invited to comment on the study design and were not consulted to develop patient relevant outcomes. Patients were not invited to contribute to the writing or editing of this document for readability or accuracy [48].

\section{Discussion}

PFP is one of the most common causes of AKP. The difficulty in treating patients with PFP is clear as many have recurrence or persisting symptoms long after treatment. Major factors for this could be the unclear etiology of PFP as well as the pains source and the subgroups of PFP patients. Hip and knee exercises have been found to be appropriate for patients with PFP and it has been shown that protocols using both hip and knee exercises seem more efficient in reducing symptoms than only knee exercises. BFR training, although not a new method of exercise, is relatively new in studies for PFP patients. This is the first study comparing hip and knee focused exercises versus hip and knee focused exercises with the use of BFR training, in adults with PFP in Cyprus. One other study has addressed the use of BFR training in PFP patients but did not include hip exercises [23]. Our study results will indicate any difference in immediate and short-term outcomes in this population regarding these two types of exercise. Additionally, it will be interesting to investigate for any muscle changes that may occur to muscle groups trained above the level of the cuff. Our study is not without limitations. One limitation could be the short follow up at 2 months due time constraints, nevertheless our goal is to assess short term outcomes. Another limitation could be considered as the level of blinding, as patients could not be blinded to treatment. Finally, we will not include a placebo or non-treatment control group due to ethical issues. Outcome measures of this study (TSK, PCS, MPFFA-DSDT, HHD) will be tested for intra rater reliability prior to the main study in order to ensure the assessor (AC) will have consistency during assessment.

\section{Acknowledgment}

We would like to acknowledge the future contributions of Dr. Pantelis Xenophontos for agreeing to screen all participants and Dr. Giangos Lavranos for agreeing to overlook participants safety. Many thanks to Ioanna Stylianou and Rolandi Christodoulidou, the two physiotherapists who will be in charge of treatments. Lastly, we acknowledge the help of our fourth-year undergraduate physiotherapy students, Anastasios Pouikkas, Doros Constantinou, Paraskevi Neophytou, Savvas Charalambous and Sophia Chrysanthou who will assist the two physiotherapists in charge of treatments.

\section{References}

1. Crossley KM, Stefanik JJ, Selfe J, Collins NJ, Davis IS, et al. (2016) Patellofemoral pain consensus statement from the $4^{\text {th }}$ international patellofemoral pain research retreat, Manchester. Part 1: Terminology, definitions, clinical examination, natural history, patellofemoral osteoarthritis and patient-reported outcome measures. Br J Sports Med 50(14): 839-843.

2. Smith BE, Selfe J, Thacker D, Hendrick P, Bateman M, et al. (2018) Incidence and prevalence of patellofemoral pain: A systematic review and meta-analysis. PLoS One 13(1): e0190892.

3. Waryasz GR, Mcdermott AY (2008) Patellofemoral pain syndrome (PFPS): A systematic review of anatomy and potential risk factors. Dyn Med 7: 9. 
4. Davis IS, Powers CM (2010) Patellofemoral pain syndrome: Proximal, distal, and local factors-an international research retreat. J Orthop Sports Phys Ther 40(3): A1-A16.

5. Witvrouw E, Callaghan MJ, Stefanik JJ, Noehren B, Bazett JDM, et al. (2014) Patellofemoral pain: Consensus statement from the 3rd international patellofemoral pain research retreat held in vancouver, September 2013. Br J Sports Med 48(6): 411-414.

6. Cook C, Mabry L, Reiman MP, Hegedus EJ (2012) Best tests/clinical findings for screening and diagnosis of patellofemoral pain syndrome: A systematic review. Physiotherapy 98(2): 93-100.

7. Nunes GS, Luiz E, Hors M, Noronha M, Santos GM (2013) Clinical test for diagnosis of patellofemoral pain syndrome: Systematic review with meta-analysis. Phys Ther Sport 14(1): 54-59.

8. Papadopoulos K, Stasinopoulos D, Dimitar G (2015) A systematic review of reviews in patellofemoral pain syndrome. Exploring the risk factors, diagnostic tests, outcome measurements and exercise treatment. Open Sport Med J 9: 7-17.

9. Collins NJ, Barton CJ, Middelkoop MV, Callaghan MJ, Rathleff MS, et al. (2018) Consensus statement on exercise therapy and physical interventions (orthoses, taping and manual therapy) to treat patellofemoral pain: Recommendations from the $5^{\text {th }}$ international patellofemoral pain research retreat, Gold coast, Australia, 2017. Br J Sport Med 52(18): 1170-1178.

10. Fukuda TY, Melo WP, Zaffalon BM, Rossetto FM, Magalhães E, et al. (2012) Hip posterolateral musculature strengthening in sedentary women with patellofemoral pain syndrome: A randomized controlled clinical trial with 1-year follow-up. J Orthop Sport Phys Ther 42(10): 823-830.

11. Fukuda TY, Rossetto FM, Magalhães E, Bryk FF, Lucareli PR, et al. (2010) Short-term effects of hip abductors and lateral rotators strengthening in females with patellofemoral pain syndrome: A randomized controlled clinical trial. J Orthop Sport Phys Ther 40(11): 736-742.

12. Khayambashi K, Mohammadkhani Z, Ghaznavi K, Lyle MA, Powers CM (2012) The effects of isolated hip abductor and external rotator muscle strengthening on pain, health status, and hip strength in females with patellofemoral pain: A randomized controlled trial. J Orthop Sport Phys Ther 42(1): 22-29.

13. Khayambashi K, Fallah A, Movahedi A, Bagwell J, Powers C (2014) Posterolateral hip muscle strengthening versus quadriceps strengthening for patellofemoral pain: A comparative control trial. Arch Phys Med Rehabil 95(5): 900-907.

14. Ferber R, Bolgla L, Earl BJE, Emery C, Hamstra WK (2015) Strengthening of the hip and core versus knee muscles for the treatment of patellofemoral pain: A multicenter randomized controlled trial. J Athl Train 50(4): 366-377.

15. Bolgla LA, Earl BJ, Emery C, Hamstra WK, Ferber R (2016) Pain, function, and strength outcomes for males and females with patellofemoral pain who participate in either a hip/core- or knee-based rehabilitation program. Int J Sports Phys Ther 11(6): 926-35.

16. Baldon RM, Serrão FV, Scattone SR, Piva SR (2014) Effects of functional stabilization training on pain, function, and lower extremity biomechanics in women with patellofemoral pain: A randomized clinical trial. J Orthop Sport Phys Ther 44(4): 240-251.

17. (2009) American college of sports medicine position stand. Progression models in resistance training for healthy adults. Med Sci Sports Exerc 41(3): 687-708.

18. Loenneke JP, Wilson GJ, Wilson MJ (2010) A mechanistic approach to blood flow occlusion. Int J Sport Med 31(1): 1-4.

19. Fahs CA, Loenneke JP, Thiebaud RS, Rossow LM, Kim D, et al. (2015) Muscular adaptations to fatiguing exercise with and without blood flow restriction. Clin Physiol Funct Imaging 35(3): 167-176.
20. Ladlow P, Coppack RJ, Dharm DS, Conway D, Sellon E, et al. (2018) Lowload resistance training with blood flow restriction improves clinical outcomes in musculoskeletal rehabilitation: A single-blind randomized controlled trial. Front Physiol 9: 1269.

21. Scott BR, Loenneke JP, Slattery KM, Dascombe BJ (2014) Exercise with blood flow restriction: An updated evidence-based approach for enhanced muscular development 45(3): 313-325.

22. Korakakis V, Whiteley R, Epameinontidis K (2018) Blood flow restriction induces hypoalgesia in recreationally active adult male anterior knee pain patients allowing therapeutic exercise loading. Phys Ther Sport 32: 235-243.

23. Giles L, Webster KE, McClelland J, Cook JL (2017) Quadriceps strengthening with and without blood flow restriction in the treatment of patellofemoral pain: A double-blind randomised trial. Br J Sports Med 51(23): 1688-1694.

24. Bowman EN, Elshaar R, Milligan H, Jue G, Mohr K, et al. (2019) Proximal, distal, and contralateral effects of blood flow restriction training on the lower extremities: A randomized controlled trial. Sport Health 11(2): 149-156.

25. Korakakis V, Whiteley R, Giakas G (2018) Low load resistance training with blood flow restriction decreases anterior knee pain more than resistance training alone. A pilot randomised controlled trial. Phys Ther Sport 34: 121-128.

26. Chan AW, Tetzlaff JM, Altman DG, Laupacis A, Gøtzsche PC, et al. (2013) SPIRIT 2013 statement: Defining standard protocol items for clinical trials. Ann Intern Med 158(3): 200-207.

27. Fox A, Ferber R, Saunders N, Osis S, Bonacci J (2018) Gait kinematics in individuals with acute and chronic patellofemoral pain. Med Sci Sports Exerc 50(3): 502-509.

28. Papathanasiou G, Georgoudis G, Papandreou M, Spyropoulos P, Georgakopoulos D, et al. (2009) Reliability measures of the short International Physical Activity Questionnaire (IPAQ) in greek young adults. Hell J Cardiol 50(4): 283-294.

29. Manske RC, Davies GJ (2016) Examination of the patellofemoral joint. Int J Sports Phys Ther 11(6): 831-853.

30. https://www.sealedenvelope.com/simple-randomiser/v1/lists.

31. Suresh K (2011) An overview of randomization techniques: An unbiased assessment of outcome in clinical research. J Hum Reprod Sci 4(1): 8-11.

32. Campanholi JN, Cedin L, Dato CC (2015) A single session of testing for One Repetition Maximum (ORM) with eight exercises is trustworthy. J Exerc Physiol online 18.

33. Gail S, Künzell S (2014) Reliability of a 5-repetition maximum strength test in recreational athletes. Dtsch Z Sportmed 65: 314-317.

34. Haff G, Triplett T (2016) Essentials of strength training and conditioning fourth edition.

35. Hughes L, Jeffries O, Waldron M, Rosenblatt B, Gissane C, et al. (2018) Influence and reliability of lower-limb arterial occlusion pressure at different body positions. Peer J 6: e4697.

36. Kujala UM, Jaakkola LH, Koskinen SK, Taimela S, Hurme M, et al. (1993) Scoring of patellofemoral disorders. Arthroscopy 9(2): 159-163.

37. Crossley KM, Bennell KL, Cowan SM, Green S (2004) Analysis of outcome measures for persons with patellofemoral pain: which are reliable and valid? Arch Phys Med Rehabil 85(5): 815-822.

38. Papadopoulos C, Constantinou A, Cheimonidou AZ, Stasinopoulos D (2017) Greek cultural adaption and validation of the Kujala anterior knee pain scale in patients with patellofemoral pain syndrome. Disabil Rehabil 39(7): 704-708. 
39. Korakakis V, Whiteley R, Epameinontidis K (2018) Blood flow restriction induces hypoalgesia in recreationally active adult male anterior knee pain patients allowing therapeutic exercise loading. Phys Ther Sport 32: 235-243.

40. Maclachlan LR, Collins NJ, Matthews MLG, Paul WH, Vicenzino B (2017) The psychological features of patellofemoral pain: A systematic review. Br J Sports Med 51(9): 732-742.

41. Kori SH, Miller RP, Todd, DD (1990) Kinesophobia: A new view of chroni pain behaviour. Pain Management 3: 35-43.

42. Rombach I, Gray AM, Jenkinson C, Murray DW, Rivero A (2018) Multiple imputation for patient reported outcome measures in randomised controlled trials: Advantages and disadvantages of imputing at the item, subscale or composite score level. BMC Med Res Methodol 18(1): 87.

43. Domenech J, Sanchis AV, López L, Espejo B (2013) Influence of kinesiophobia and catastrophizing on pain and disability in anterior knee pain patients. Knee Surgery Sport Traumatol Arthrosc 21(7): 1562-1568.
44. Georgoudis G, Katsoulakis K, Kanellou B (2005) Cognitive assessment of chronic musculoskeletal pain: The case of patient fear of injury. Physiotherapy Topics 3: 57-66.

45. Sullivan MJL, Bishop SR, Pivik J (1995) The pain catastrophizing scale: Development and validation. 7(4): 524-532.

46. http://www.paininmotion.be/education/tools-for-clinical-practice.

47. Ophey MJ, Bosch K, Khalfallah FZ, Wijnands AMMP, Berg RB et al. (2019) The decline step-down test measuring the maximum pain-free flexion angle: A reliable and valid performance test in patients with patellofemoral pain. Phys Ther Sport 36: 43-50.

48. Maffiuletti NA (2010) Assessment of hip and knee muscle function in orthopaedic practice and research. J Bone Joint Surg Am 92(1): 220-229.

For possible submissions Click below: 